\title{
Percepção de professores e estudantes em relação ao perfil de formação do nutricionista em saúde pública ${ }^{1}$
}

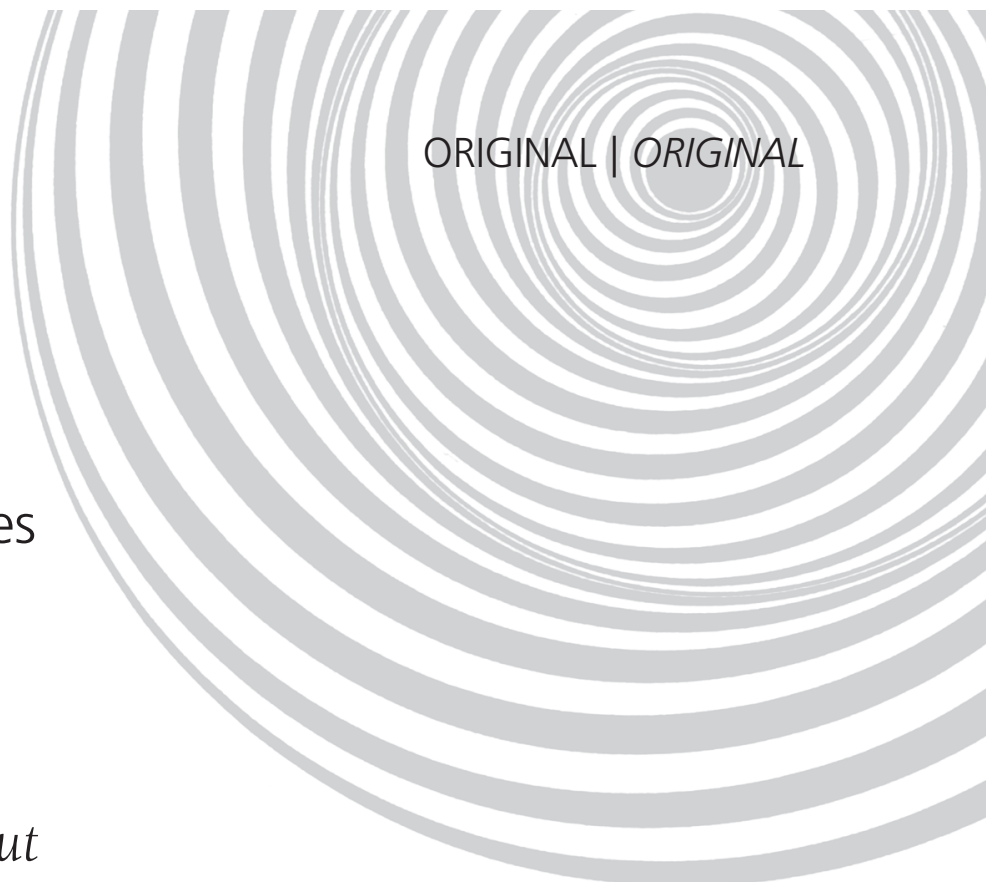

Perception of teachers and students about

the education of dieticians who

specialize in public health

Anelise Rizzolo de Oliveira PINHEIRO²

Elisabetta RECINE²

Bárbara de ALENCAR ${ }^{2}$

Andhressa Araújo FAGUNDES²

Jussara Santos de SOUSA ${ }^{2}$

Renata Alves MONTEIRO2

Natacha TORAL ${ }^{2}$

RE S U M O

\section{Objetivo}

Analisar a percepção de professores e estudantes em relação ao perfil de formação do nutricionista em saúde pública.

\section{Métodos}

A pesquisa, de abordagem qualiquantitativa, foi realizada com participantes do $21^{\circ}$ Congresso Brasileiro de Nutrição. Foi aplicado um questionário com estudantes e recém-egressos, e feito um grupo focal com professores. Foi estabelecida a análise descritiva dos dados quantitativos, e a análise qualitativa foi desenvolvida por meio da análise do discurso do sujeito coletivo.

\section{Resultados}

Dentre os estudantes, 35\% relataram ter afinidade com a área de saúde pública, e $11 \%$ dos egressos disseram atuar em saúde pública. Segundo os professores, as principais barreiras percebidas na formação do nutricionista em saúde pública foram: dificuldade de inserção do profissional na área; relação entre teoria e prática; e relação ensino e serviço de saúde.

\footnotetext{
1 Apoio: Ministério da Saúde processo (25000 270801/2008-91), convênio 667/2008-2011.

2 Universidade de Brasília, Faculdade de Ciências da Saúde, Departamento de Nutrição. Campus Universitário Darcy Ribeiro, Núcleo de Medicina Tropical e Nutrição, Sala 9C, 70910-900, Brasília, DF, Brasil. Correspondência para/Correspondence to: ARO PINHEIRO. E-mail: <anelise.unb@gmail.com>.
} 
632 ARO PINHEIRO et al.

\section{Conclusão}

Os aspectos de saúde pública na formação do nutricionista ainda são incipientes, o que evidencia a necessidade de um maior aprofundamento do tema.

Termos de indexação: Formação de recursos humanos. Nutrição em saúde pública. Nutricionista.

\section{A B S T R A C T}

\section{Objective}

This study analyzed how professors and students perceive the education of dieticians who specialize in public health.

\section{Methods}

This qualitative and quantitative study administered a questionnaire to undergraduate and graduate students and held a focal group with professors. The data collected by the questionnaire was descriptively analyzed and the contents of the focal group were analyzed according to the Discourse of the Collective Subject method.

\section{Results}

Thirty-five percent of the students mentioned some kinship with the public health field and $11 \%$ of the graduates reported working in public health. Meanwhile, the greatest barriers in public health education, according to professors, were: difficulty of finding a job in the area, the relationship between theory and practice, and the communication difficulty between teaching and health service.

\section{Conclusion}

These results indicate the need broadening and deepening the public health theme in undergraduate nutrition courses.

Indexing terms: Human resources formation. Nutrition, public health. Nutritionist.

\section{N T R O D U ÇÃ O}

O nutricionista é um profissional generalista que visa à promoção da segurança alimentar e à atenção nutricional em todas as áreas do conhecimento e atuação em que a alimentação e a nutrição sejam fundamentais para a promoção, a manutenção e a recuperação da saúde e para a prevenção de doenças de indivíduos ou grupos populacionais. De acordo com as Diretrizes Curriculares do Curso de Graduação em Nutrição' , o nutricionista atua para contribuir com a melhora da qualidade de vida, pautando-se em princípios éticos e refletindo sobre a realidade social.

A situação epidemiológica brasileira representada pela dupla carga de doenças tem exigido dos nutricionistas uma reflexão mais aprofundada de seu papel nas estratégias para reversão desse cenário. Superar problemas alimentares e nutricionais requer ações articuladas com enfoque na promoção da alimentação saudável no Sistema
Nacional de Segurança Alimentar e Nutricional $\left(\right.$ SISAN) ${ }^{2}$ e atenção nutricional no âmbito do Sistema Único de Saúde (SUS) ${ }^{3}$. Atualmente, a complexidade dos problemas alimentares e nutricionais impõe reformulações na formação dos profissionais de saúde, como o fortalecimento do conteúdo de saúde pública nos currículos dos cursos de nutrição.

Os cursos de Nutrição na América Latina tiveram seu marco inicial de fundação no Instituto Nacional de Nutrição e na Escola de Dietistas em 1933, em Buenos Aires, com apoio do médico argentino Pedro Escudero ${ }^{4}$. No Brasil, a formação de nutricionista teve início com o curso de Nutrição criado, em 1939, pelo Instituto de Higiene, atualmente Faculdade de Saúde Pública da Universidade de São Paulo 5 .

Historicamente, a instituição do II Programa Nacional de Alimentação e Nutrição (PRONAN) do Instituto Nacional de Alimentação e Nutrição (INAN) contribuiu para expansão dos cursos de 
Nutrição no Brasil. A primeira fase de expansão ocorreu de 1975 a 1981, com o aumento de 7 para 30 cursos nacionais ${ }^{4,6}$. A segunda fase de expansão (1985-2000) foi mais intensa e com predominância de participação do setor privado. Conforme citado por Vasconselos ${ }^{7}$, em 2000, existiam 106 cursos, sendo 22 públicos e 84 privados. Desde então, o processo tem evoluído quase que exclusivamente em função da criação de cursos privados. Atualmente, estão registrados no Ministério da Educação (MEC), em atividade, 379 cursos de bacharelado em Nutrição, sendo 60 de instituições de ensino superior públicas e 319 de privadas.

Esse aumento expressivo de cursos tem provocado uma reflexão do ponto de vista da qualidade do processo de formação. Quais as bases e os pressupostos pedagógicos que ancoram a organização dos currículos e projetos político-pedagógicos dos cursos de Nutrição no Brasil? Qual o perfil do profissional de Nutrição que se espera diante do novo e complexo contexto epidemiológico, político e social? Em termos de inserção do nutricionista nos campos de prática profissional, como tem se dado a relação entre o aumento de cursos e de vagas de Nutrição e a oferta de emprego, especialmente no âmbito da Nutrição em saúde pública?

Assim, o atual campo de atuação do nutricionista demanda um profissional crítico, criativo, integrador e com habilidade de trabalhar em equipe inter e multiprofissional, além de ser capaz de solucionar conflitos e compreender o SUS como cenário de práticas das ações de saúde em toda sua abrangência. O presente estudo tem o objetivo de analisar a percepção de estudantes e professores sobre o perfil de formação em Nutrição em saúde pública dos cursos públicos e privados no Brasil.

\section{M É T O D O S}

Pesquisa descritiva com abordagem qualiquantitativa. Utiliza-se a estratégia de pesquisa da triangulação, que se apoia em métodos cien- tíficos testados, adequando realidades com fundamentos interdisciplinares, permitindo o cruzamento de pontos de vista, a visão de vários informantes e o emprego de técnicas que acompanham o trabalho de investigação?.

Os sujeitos do estudo foram professores, estudantes de graduação e egressos dos cursos de Nutrição participantes do $21^{\circ}$ Congresso Brasileiro de Nutrição (CONBRAN), realizado entre os dias 26 e 29 de maio de 2010, em Joinville (SC) ${ }^{10}$. A amostra da pesquisa foi realizada por conveniência. A escolha de estudantes e egressos ocorreu por meio de convite aos que transitavam no saguão do evento. As exigências para compor a amostra foram: estar cursando pelo menos o quinto semestre de Nutrição ou ter no máximo um ano de formado.

Os professores foram convidados a partir de uma oficina intitulada "Perfil de formação do nutricionista em saúde pública". Os docentes interessados fizeram inscrição, sem ônus, para participar da oficina. Os critérios de participação foram: ser docente de universidades/faculdades ou centros universitários públicos ou privados e/ou apresentar alguma interface de trabalho com o ensino de Nutrição em saúde pública.

Os estudantes responderam a um questionário autoaplicável, com perguntas sobre o perfil de formação do nutricionista em saúde pública. O instrumento tinha três itens de múltipla escolha, uma questão aberta, 12 questões afirmativas para serem avaliadas de acordo com o grau de concordância e/ou importância e 11 questões dicotômicas. Na questão aberta, foi perguntado aos participantes o motivo de terem escolhido estudar nutrição. Nas questões de múltipla escolha, foi perguntando se os estudantes haviam participado de projetos de pesquisa ou extensão em saúde pública; os possíveis locais de atuação do nutricionista na área de saúde pública; e os fatores que interferem na atuação do profissional.

Foram propostas algumas afirmativas sobre a área de saúde pública e de Nutrição em saúde pública para os participantes avaliarem por meio de uma escala de concordância. Em relação 
à atuação do nutricionista na área de saúde pública, os participantes foram questionados quanto às habilidades e às competências do profissional nessa área, sendo pedido que atribuíssem um grau de importância de 1 (nada importante) a 5 (muito importante).

As questões dicotômicas avaliaram o contato dos participantes com publicações do Ministério da Saúde durante o curso de graduação em Nutrição.

Todos os participantes foram informados sobre os objetivos da pesquisa e assinaram um Termo de Consentimento Livre e Esclarecido. A análise descritiva dos questionários dos estudantes foi realizada com apoio dos softwares Statistical Package for the Social Sciences (SPSS) 17.0 e Excel 2000. Os questionários tiveram dupla digitação.

O grupo focal realizado com os professores que participaram da oficina de trabalho foi estruturado a partir de um roteiro orientador no qual foram abordadas questões referentes às habilidades, às competências e ao perfil em nutrição em saúde pública em que o futuro profissional está sendo formado. Um facilitador coordenou o processo e dois relatores registraram os pontos centrais. A dinâmica de conversação foi gravada e, posteriormente, as falas dos participantes foram transcritas e analisadas.

Para análise e interpretação dos dados, utilizou-se a técnica do Discurso do Sujeito Coletivo (DSC), procedimento metodológico de natureza qualiquantitativa, utilizado para expressar a manifestação do pensamento de um sujeito coletivo na forma de discursos. O DSC une os discursos semelhantes e complementares dos sujeitos em um único, que representa a manifestação do pensamento do grupo em relação a um tema específico ${ }^{11}$.

Para elaborar o DSC, utilizam-se figuras metodológicas: Expressões-Chave (E-Ch), Ideias Centrais (IC), Ancoragem (AC) e Discurso do Sujeito Coletivo. As E-Ch são trechos selecionados do material verbal de cada depoimento que melhor descrevem seu conteúdo. As IC são fórmulas sintéticas que descrevem o(s) sentido(s) presente(s) nos depoimentos de cada resposta e também nos conjuntos de respostas de diferentes indivíduos, que apresentam sentido semelhante ou complementar. As AC são fórmulas sintéticas que indicam a teoria, o pressuposto, a corrente de pensamento, o "pano de fundo" do conhecimento que o sujeito compartilha e aceita de uma maneira natural para representar um dado fenômeno da realidade ${ }^{11}$. A análise das falas teve apoio do software QUALIQUANT ${ }^{\circledR}$.

O estudo foi aprovado pelo Comitê de Ética em Pesquisa da Faculdade de Saúde da Universidade de Brasília, Protocolo nº 097/09 em 16 de setembro de 2009.

\section{RESULTADOS}

Dos 197 indivíduos que participaram da pesquisa quantitativa, $178(90 \%)$ eram estudantes e os demais recém-formados, sendo $98 \%$ do sexo feminino e $90 \%$ com idade entre 19 e 24 anos (Média - $M=22$, Desvio-Padrão - DP=4,03) (Figura 1). A região mais repre-sentada foi a Sul, com $65 \%$ dos participantes, seguida pela Sudeste, com $24 \%$.

Entre os estudantes, $68 \%$ eram de faculdades particulares e $42 \%$ cursavam o $7^{\circ}$ semestre. Os demais se distribuíam entre $05^{\circ}$ semestre

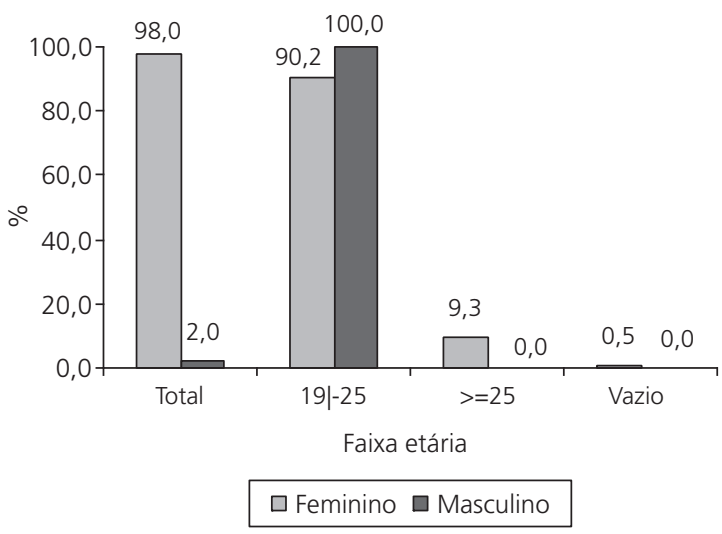

Figura 1. Distribuição por sexo dos estudantes participantes do estudo sobre percepção de estudantes sobre o perfil de formação em saúde pública.

Fonte: Congresso Brasileiro de Nutrição $(2010)^{10}$. 
(26\%), $8^{\circ}$ semestre $(14 \%), 6^{\circ}$ semestre $(11 \%)$ e $9^{\circ}$ semestre $(6 \%)$. Quando questionados a respeito de sua afinidade com alguma área da Nutrição, 92\% afirmaram ter afinidade por alguma área, sendo a principal a área de Nutrição Clínica (54\%). Nutrição em Saúde Pública foi a segunda de maior interesse, com 35\% dos respondentes. A área de Gestão de Serviços de Alimentação é a que desperta menor interesse, sendo apontada por $12 \%$ dos respondentes.

Dos participantes egressos, $74 \%(n=14)$ haviam concluído seus cursos em faculdades públicas. Aproximadamente $68 \%$ tinham entre 5 e 6 meses de formados e $58 \%$ relataram cursar pós-graduação: 37\% especialização, 5\% mestrado e residência.

Quanto à área de atuação, 63\% relataram atuar na Nutrição, sendo $21 \%$ na área de Nutrição Clínica, 16\% em Gestão de Serviços de Alimentação e 11\% na área de Saúde Pública; 16\% referiram atuar em outras áreas, como Alimentação Escolar, Indústria de Alimentos, Gastronomia, Docência e Marketing.

\section{Perfil de formação do nutricionista em saúde pública}

Do total da amostra entrevistada, 56\% afirmaram ter participado de algum curso de extensão durante a graduação, e 3\% afirmaram que a faculdade não oferecia cursos de extensão.

Quando questionados sobre os fatores que interferem na atuação do nutricionista, 58\% afirmaram a possibilidade de ascensão profissional como um fator determinante, $47 \%$ alegaram que a potencialidade para mudar a realidade interfere na atuação e $41 \%$ citaram a oferta de emprego.

Quanto ao local onde os nutricionistas podem atuar, 96\% responderam Unidades Básicas de Saúde, Núcleos de Apoio à Saúde da Família e Estratégia Saúde da Família; 84\% escolas e creches, $62 \%$ em hospitais e $67 \%$ no governo.

Na assertiva sobre a área de saúde pública ser ampla e ficar difícil para o profissional definir seu papel, 34\% dos respondentes concordaram parcialmente e $23 \%$ discordaram totalmente. Quase metade (46\%) concordou totalmente que a área de saúde pública é estratégica para a ação do nutricionista. Sobre o papel do nutricionista ser principalmente de educador em saúde, 50\% concordaram totalmente (Figura 2).

Quando questionados sobre o conhecimento das publicações disponibilizadas pelo Ministério da Saúde na área de Nutrição em Saúde Pública, 88\% afirmaram 'conhecer' o Guia Alimentar da População Brasileira, seguido do Protocolo da Vigilância Alimentar e Nutricional

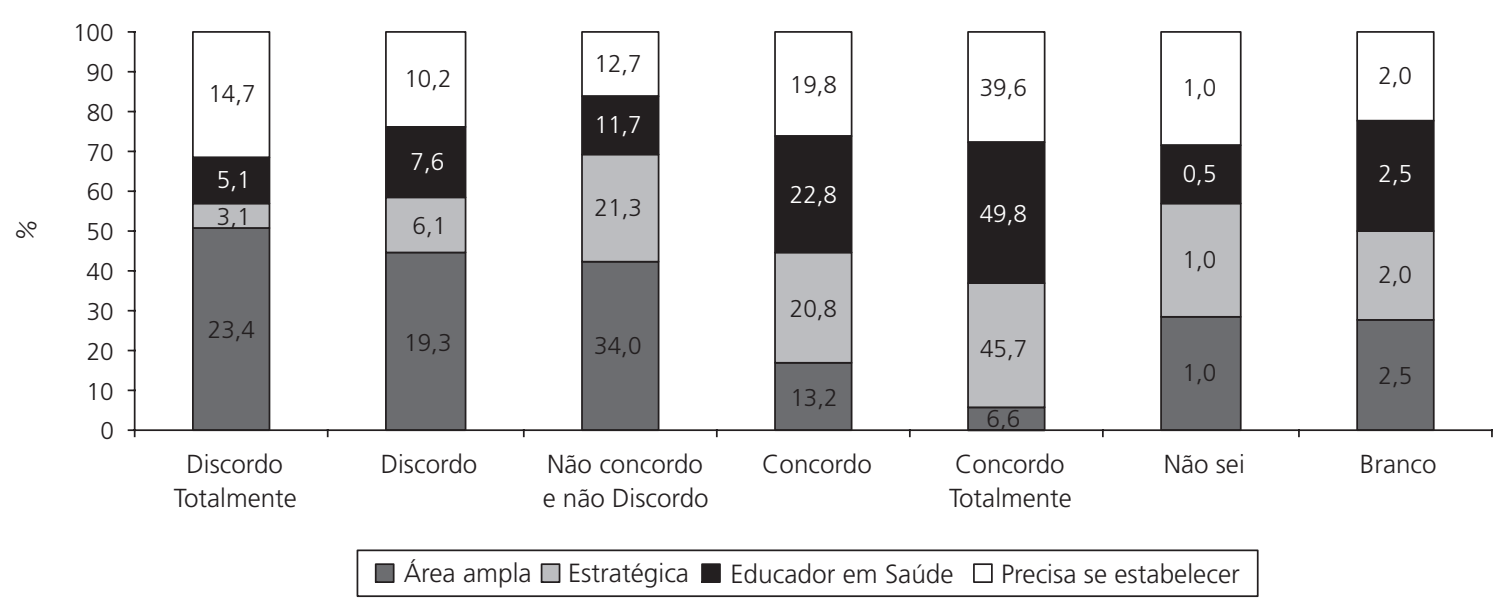

Figura 2. Opinião dos estudantes de nutrição sobre as perspectivas e possibilidades da área de nutrição em saúde pública. Fonte: Congresso Brasileiro de Nutrição $(2010)^{10}$. 
(SISVAN) e da Política Nacional de Alimentação e Nutrição (PNAN), ambos conhecidos por $80 \%$ dos respondentes. Entre os que estudaram essas publicações durante o curso de graduação, $42 \%$ relataram ter estudado a Matriz de Ações de Nutrição na Atenção Básica e as Portarias dos NASF n 154 de 2008 e $n^{\circ} 424$ de 2009. A média de publicações estudadas foi de seis referências e de 2,5 livros conhecidos por respondente.

Ainda sobre a atuação do nutricionista na área de saúde pública, os participantes foram questionados quanto às habilidades e às competências do profissional nessa área. Foram considerados "muito importantes" o trabalho em equipe $(95,9 \%)$, a atenção individual $(71,2 \%)$, a atenção em grupos $(87,2 \%)$, atividades de coordenação e execução de programas $(86,3 \%)$, realizar diagnósticos alimentares (88,8\%), avaliar a situação de segurança alimentar do grupo $(91,3 \%)$, realizar ações de promoção de alimentação saudável (94,9\%), atuar na vigilância nutricional $(83,7 \%)$ e, ainda, atuar em pesquisa (76,3\%) (Figura 3).

Em relação ao motivo da escolha do curso de Nutrição, dentre os 197 estudantes que responderam, a maioria $(42,6 \%)$ afirmou ter escolhido por interesse pessoal e afinidade, seguidos da opção Promoção da Saúde (33,0\%). Escolha devido a oportunidades profissionais foi feita por $9,6 \%$ dos participantes.

\section{Professores e o processo de formação profissional}

O grupo focal com os professores contou com a participação de nove indivíduos: quatro docentes de instituições de ensino superior públicas, quatro docentes de instituições de ensino particulares e um nutricionista fiscal do Conselho Federal de Nutricionistas da Região Sudeste. Com exceção de um participante (11\%), todos os outros (89\%) eram do sexo feminino. Quanto à distribuição por região, o percentual maior foi de professores da Região Sudeste: $50 \%$ eram provenientes dos estados do Rio de Janeiro, Espírito Santo e Minas Gerais. Por fim, dois participantes eram originários da Região Centro-Oeste e três da Região Nordeste.

$\mathrm{Na}$ análise do grupo focal dos professores, é possível estabelecer algumas reflexões sobre os aspectos de amplitude e de abrangência em relação à área de Saúde Pública.

Alguns dizem que gostam de saúde pública, mas como o perfil do profissional é muito estimulado pelo mercado, se ele está voltado para Administração de Serviços de Alimentação (ASA), ou para clínica, é lá que o aluno vai atuar. Então ele pensa ... que saúde pública não é sua área. Ele vai para o mercado e, mesmo gostan-

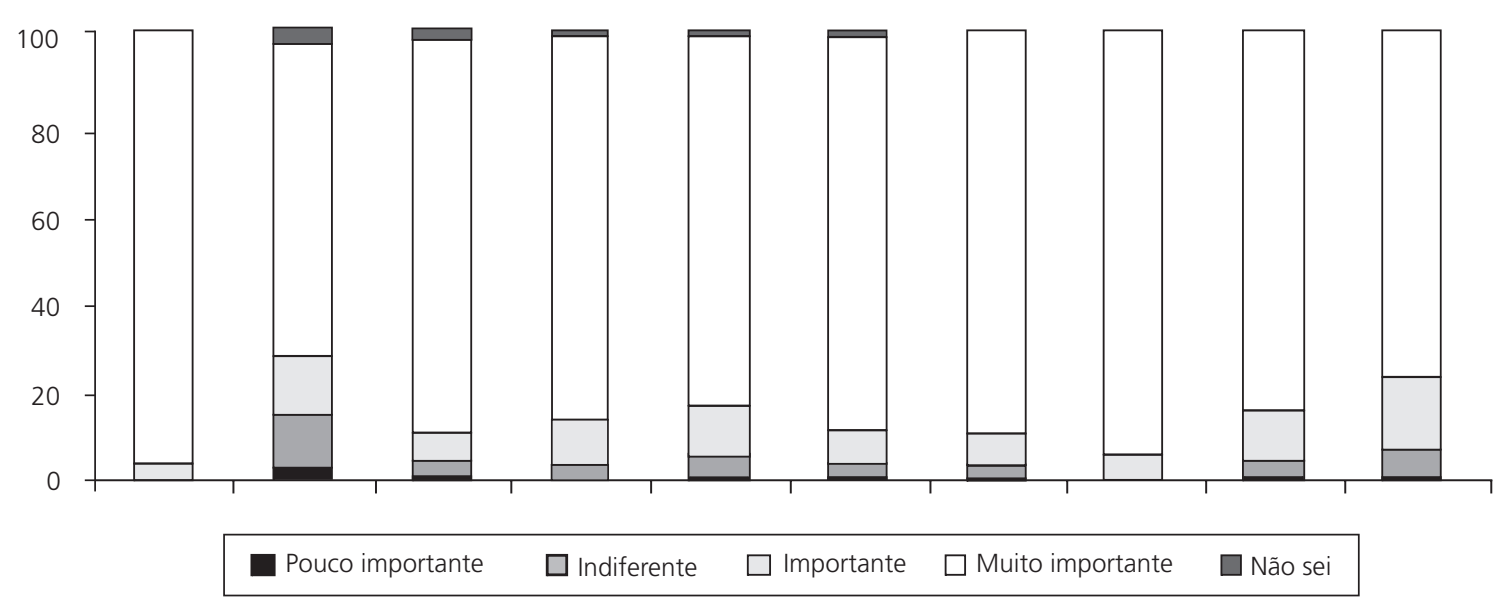

Figura 3. Competências ou responsabilidades do profissional nutricionista na área de saúde pública. 
do da saúde pública, ele terá paixão por aquilo com que irá trabalhar... . Um dos elementos que dificulta a realidade é o não cumprimento da (ou "na"?) prática. Tudo o que está no papel não é cumprido na prática... . Quando o aluno se forma ele não tem interesse em saúde pública, pois não se vê trabalhando na área... . Quando eles pensam em trabalhar em saúde pública, pensam somente em cursos da prefeitura para merenda escolar (Discurso do Sujeito Coletivo - Grupo Focal).

Para os professores no grupo focal, as possibilidades de inserção do nutricionista em nutrição social são referidas mais junto ao Programa Nacional de Alimentação Escolar (PNAE) do que nos serviços de saúde/atenção básica e saúde da família.

A questão norteadora do debate foi o perfil do nutricionista em Saúde Pública que está sendo formado nas Instituições de Ensino Superior. As ideias centrais mais frequentes na discussão foram: inserção do nutricionista em Saúde Pública (32\%), relação entre teoria e prática (28\%), e relação entre ensino e serviço de saúde (12\%) (Figura 4).
Identificaram-se, nesta abordagem, algumas habilidades e competências esperadas para o perfil de formação dos alunos:

Uma das competências seria a reflexão. O aluno precisa refletir sobre a realidade, tem de ser capaz de identificar seu papel no contexto em que está inserido e refletir sobre como a nutrição se aplica nesse contexto. Outra habilidade é a de ser proativo, de propor intervenções, identificar em uma determinada situação o problema, e propor uma solução... . o aluno tem de pensar nos diagnósticos mais amplamente, não bastando fazer só o diagnóstico clínico. Tem de fazer o diagnóstico cultural, social e econômico. Ele tem que conhecer a dinâmica social do local, a capacidade de organização dessa sociedade para planejar sua ação..., pensando de forma multidisciplinar. Ele tem que fazer o diagnóstico, planejar, executar e avaliar a ação (Discurso do Sujeito Coletivo - Grupo Focal).

Quando foi abordada a relação teoria e prática nos cursos de Nutrição, os participantes destacaram o problema da linearidade do pro-

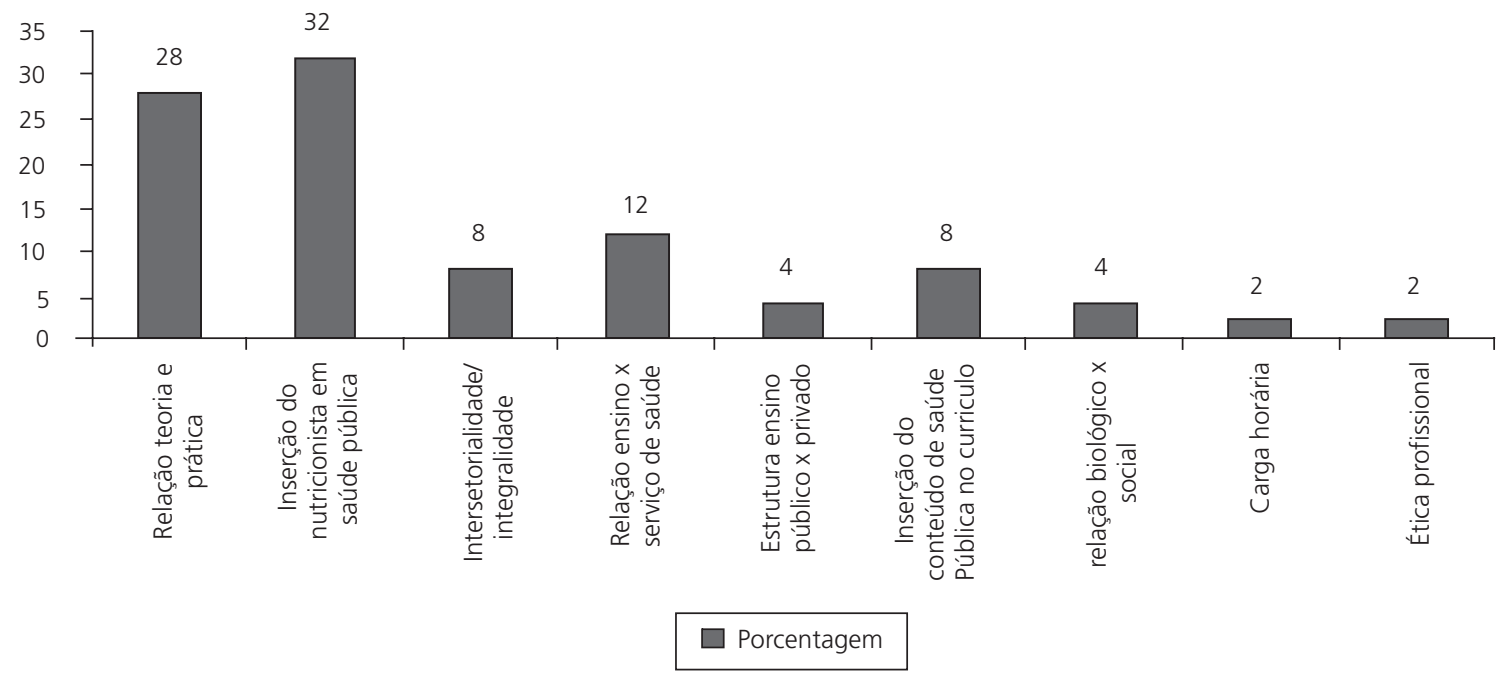

Figura 4. Distribuição de frequência das ideias centrais na discussão do grupo focal com professores, sobre o perfil do estudante de nutrição no processo de formação em saúde pública. 
cesso de ensino, que visa primeiro abordar aspectos teóricos, deixando a prática para o final do curso.

Um grande problema é que a prática ocorre mais para o final... . O aluno vai até o quinto período com disciplinas muito biológicas, e por volta do sexto semestre ele encontra as disciplinas de social... . Nesse período se tenta romper com a visão biológica... . Quando ele vai para o estágio curricular no último período, acaba descobrindo melhor a área de saúde pública ... . Ele não aprende saúde pública, pois não tem motivação para fazer a disciplina desde o início do curso (Discurso do Sujeito Coletivo - Grupo Focal).

Outro aspecto abordado pelos sujeitos participantes foi o caráter biologicista dos cursos de Nutrição.

A Nutrição não é só biologia. Você lida com um ser humano que vive em sociedade, que está em um ambiente físico, muitas vezes degradado. Então trazer para a sala de aula essa discussão seria interessante (Discurso do Sujeito Coletivo - Grupo Focal).

Para o grupo debatedor, a perspectiva da integralidade no âmbito da gestão tem maior potencialidade de concretização em municípios de pequeno porte:

... em locais com uma população menor, como em pequenos municípios, fica mais fácil articular várias ações de Nutrição, dá para organizar os programas, articular coordenação de atenção básica... . Quando o município é grande, há uma dificuldade de integração (Discurso do Sujeito Coletivo - Grupo Focal).

No entanto, a dimensão da integralidade no processo de formação do nutricionista reflete novamente a questão da fragmentação da estrutura curricular:

... se as escolas de Nutrição buscassem construir práticas integradas, o aluno seria capaz de trabalhar saúde pública com outro viés..., fazer com que ele seja capaz de trabalhar criticamente, para que ele busque apoio junto a instituições, para que ele possa explorar espaços públicos, pensar em políticas públicas (Discurso do Sujeito Coletivo - Grupo Focal).

A discussão do grupo também indicou questões sobre a relação das universidades com os serviços de saúde, ou seja, como o processo de formação e capacitação dos estudantes tem participado e se relacionado com o campo de práticas da Nutrição nos serviços que recebem estagiários. Essa dimensão esbarra em outras questões centrais do debate: a relação teoria e prática e a inserção do nutricionista na Atenção Básica de Saúde-SUS. A ausência do profissional demanda estratégias das instituições de formação acadêmica para propiciar a vivência prática dos estudantes, e, nesse sentido, muitos relatos destacaram que o próprio discente acaba assumindo a função do profissional no período da atividade prática curricular, e que, após sua finalização, a oferta do serviço desaparece, não garantindo à comunidade assistida continuidade na atenção nutricional.

\section{I S C U S S Ã O}

Parece ser consenso entre os docentes e educadores envolvidos com o tema de educação e saúde a necessidade de mudanças no processo de formação. Nesse sentido, visando a novos campos de prática para formação interdisciplinar, o Ministério da Saúde instituiu o Programa Nacional de Reorientação da Formação Profissional em Saúde (Pró-Saúde) ${ }^{12}$, que visa a contribuir para o fortalecimento das ações de atenção básica de saúde do SUS.

A Atenção Básica é definida como um conjunto de ações de saúde, no âmbito individual e coletivo, que abrange a promoção e a proteção da saúde, a prevenção de agravos, o diagnóstico, o tratamento, a reabilitação e a manutenção da saúde. É desenvolvida por meio do exercício de 
práticas democráticas e participativas sob a forma de trabalho em equipe, dirigidas a populações de territórios bem delimitados, considerando a dinamicidade existente no território em que vivem essas populações. "Orienta-se pelos princípios da universalidade, da acessibilidade e da coordenação do cuidado, do vínculo e da continuidade, da integralidade, da responsabilização, da humanização, da equidade e da participação social" ${ }^{\text {. }}$.

A política nacional de atenção básica a Estratégia de Saúde da Família (ESF) é estruturante dos sistemas de saúde e coordenadora do cuidado, articulado numa rede de serviços de referência e contrarreferência. A equipe mínima de saúde da família é composta por médico, enfermeiro, técnicos de enfermagem e agentes comunitários, integrando também uma equipe de saúde bucal. Para ampliar e fortalecer sua atuação, foram criados os Núcleos de Apoio à Saúde da Família (NASF), incorporando outros perfis profissionais em equipes matriciais, como o nutricionista ${ }^{13}$, que têm suas ações e programas, no âmbito da atenção básica - SUS, articulados pela PNAN, que também congrega o conjunto de propósitos e diretrizes para promoção da alimentação saudável no Brasil ${ }^{14}$.

\section{Pesquisa com alunos e recém-egressos dos cursos de nutrição}

Os resultados verificados na presente pesquisa apresentaram uma predominância de estudantes e professores de nutrição da Região Sul (64\%), provavelmente devido à localização geográfica do evento: o Estado de Santa Catarina. Mais da metade dos recém-egressos (58\%) relataram cursar algum tipo de pós-graduação ou especialização: resultados semelhantes aos encontrados no estudo conduzido por Gambardella et al. ${ }^{15}$ com recém-egressos de Nutrição: 37,1\% dos participantes referiram continuar estudando após a conclusão do curso, e, dentre eles, 29,2\% afirmaram estar realizando alguma especialização na área de Nutrição. Em relação à atuação, 63\% dos entrevistados disseram que atuam na área de Nutrição, a maioria na área de nutrição clínica, seguida pelas áreas de Produção e Saúde Pública.

Ao estudar o perfil profissional dos nutricionistas egressos de uma universidade paulista, Gambardella et al. ${ }^{15}$ observaram que 36,6\% atuavam em Nutrição Clínica, 31,0\% em unidades de alimentação e nutrição e 7,0\% em Saúde Pública. Em estudo conduzido com recém-egressos também de uma universidade paulista, Miranda et al. ${ }^{16}$ encontraram que $85,5 \%$ dos egressos estavam atuando na área. Dentre esses, 56,6\% na área de Nutrição Clínica e 53,8\% na área de Alimentação Coletiva; 7,2\% atuavam na área de Saúde Pública e outros 7,2\% em outras áreas como Nutrição Esportiva e Marketing.

A maioria dos participantes ( $56 \%$ ) relatou ter participado de cursos de extensão durante a graduação. Ações de extensão executadas concomitantemente à graduação são de grande importância para o aluno por serem uma atividade em que se pode construir conhecimentos, aliando o aprendizado teórico obtido em sala de aula ao contato prático com a realidade e portanto, complementando e ampliando seu aprendizado.

Segundo Goulart ${ }^{17}$, é necessário que as atividades de extensão façam parte do processo de ensino, e que os currículos tenham flexibilidade para propiciar a articulação entre ensino e extensão.

Em relação ao perfil de formação do nutricionista, 50\% dos participantes disseram que o nutricionista tem como papel principal o de educador em saúde. Bandulk et al. ${ }^{18}$, em estudo conduzido na cidade de São Paulo com estudantes de Nutrição, enfatizou o papel de educador do nutricionista. Para os estudantes, a educação alimentar e nutricional influencia diretamente a qualidade de vida das pessoas.

Se, por um lado, a prática profissional do nutricionista em saúde pública encontra limites em termos de inserção profissional, a dimensão de "ser educador" pode ser um fator de influência na percepção dos estudantes sobre o campo da saúde pública. 
Em relação à escolha do curso superior, 33,0\% dos entrevistados escolheram a Nutrição por ela ser capaz de promover a saúde. Em contrapartida, no estudo conduzido por Portronieri et al. ${ }^{19}$, um dos motivos da escolha do curso de Nutrição por $77,8 \%$ dos entrevistados foi o interesse em temas relacionados à Saúde e à Alimentação.

\section{Os professores e o processo de formação de estudantes de nutrição}

No grupo focal realizado com os professores, foram levantadas questões referentes às possibilidades de atuação do nutricionista em Nutrição Social. De acordo com esse grupo, as possibilidades de inserção do nutricionista em Nutrição Social são maiores junto ao Programa Nacional de Alimentação Escolar (PNAE).

O PNAE, nos últimos anos, obteve um avanço inegável no marco das políticas públicas de alimentação e nutrição. Com o apoio do Conselho Nacional de Segurança Alimentar e Nutricional (CONSEA), seu arcabouço legal foi atualizado e recebeu fortes estímulos técnicos e políticos. De acordo com os pressupostos do Direito Humano à Alimentação Adequada e com o enfoque da promoção da alimentação saudável, é um dos programas chaves da PNAN ${ }^{20}$. Além disso, adquiriu o status de lei, o que ampliou seu marco regulatório quando definiu a obrigatoriedade do nutricionista como responsável técnico das ações do Programa em nível municipal.

Outra possibilidade de atuação do nutricionista em saúde pública são os Núcleos de Apoio à Saúde da Família (NASF), uma iniciativa mais recente do Ministério da Saúde, ainda em fase de implantação na maioria dos municípios brasileiros. A presença de nutricionista está prevista em todos os tipos de NASF: 1, 2 e 3. Em maio de 2010, os nutricionistas estavam presentes em $74,5 \%$ das 1157 equipes de NASF inseridas no Cadastro Nacional de Estabelecimentos de Saúde $(\mathrm{CNES})^{21}$.

Em relação às habilidades e competências esperadas para a formação dos alunos, o grupo destacou a necessidade de abordagens de ensino capazes de articular metodologias ativas e aplicadas à realidade. Além de identificar questões, é preciso contextualizar os diagnósticos, refletir e discutir de maneira participativa para, então, planejar alternativas e soluções. Além disso, é fundamental desenvolver a capacidade de resolver problemas em todas as etapas do processo.

De acordo com Santos ${ }^{22}$, em relação ao processo de formação dos profissionais de saúde, em particular dos nutricionistas, as evoluções pedagógicas e educacionais demandam mudanças a fim de se formar um profissional que se integre melhor ao mundo do ensino e do trabalho numa perspectiva multiprofissional. A introdução de metodologias ativas de aprendizado junto à diversificação de cenários de práticas ao longo de todo processo de ensino-aprendizagem são questões centrais para alterar a estrutura dos currículos atuais. Porém, a tradução dessas demandas em atividades e conteúdo ainda é um desafio dos cursos da saúde.

No aspecto da relação entre teoria e prática, percebe-se que a maioria dos currículos da área da saúde orienta-se pela concepção de construção de conhecimento positivista, que compreende a organização dos processos de ensino de "forma linear, do teórico para o prático e do ciclo básico para o profissionalizante" (online). Essa abordagem pressupõe que os conteúdos devam ser ministrados previamente, a fim de que o estudante seja capaz de "dominar" a teoria e, então, aplicá-la na prática, não incorporando a prática como um espaço pedagógico de ensino e aprendizagem ${ }^{21,22}$.

Para Minayo ${ }^{9}$, a teoria positivista tem na sua forma derivativa o funcionalismo sociológico, a corrente de pensamento mais hegemônica em termos de influência e vigor na produção intelectual de saúde pública, que reproduz, historicamente, práticas autoritárias e impositivas. $\mathrm{Na}$ prática médica e dos cursos de saúde em geral, e nas suas relações com a sociedade, o positivismo se expressa na concepção do processo saúde-doença como um fenômeno apenas biológico e individual, na excessiva valorização de tecnologias, na dominação de grupos de profissionais cor- 
porativos com destaque para a categoria dos médicos em relação aos outros campos de conhecimento, na utilização de outros saberes como antropologia e sociologia apenas de forma pontual para produzir informativos, questionários e conceitos básicos, no menosprezo pelos valores culturais e, por fim, em uma postura autoritária da categoria dos médicos no tratamento com os outros profissionais da área da saúde.

Os participantes também destacaram o caráter biologicista de grande parte dos cursos de Nutrição no País. Esse aspecto pode ser explicado pelas estruturas curriculares dos cursos de Nutrição que têm um viés de concepção baseado no modelo Flexneriano, que serviu de referência para a implantação das escolas médicas e dos cursos de saúde em geral, com base em um modelo de saúde biologicista, com ênfase nas ciências básicas e na prática curativa22,23.

Outro problema levantado foi a questão da formação diferenciada do profissional, voltada para atenção em saúde em resposta às demandas sociais.

Historicamente a formação crítico-reflexiva, que permite um pensamento autônomo para compreensão da totalidade do sujeito, assumindo a formação como um processo interativo e dinâmico, esbarra nas estruturas curriculares como limite de possibilidades ${ }^{24}$. Nos últimos anos, contudo, especialmente na área da saúde, têm tido destaque debates sobre estratégias de ensino que ampliem a perspectiva pedagógica e que permitam a incorporação de pesquisa e extensão, associadas a metodologias ativas no processo de formação do ensino superior.

A complexidade do processo de produção social da saúde exige que a atenção à saúde repense sua práxis no sentido da consolidação efetiva dos princípios do SUS. Mudar o modelo de atenção à saúde em busca da integralidade implica o desenvolvimento de um trabalho multiprofissional, com abordagem interdisciplinar e intersetorial.

A integralidade é eixo importante para apoiar as mudanças na formação de profissionais da área e desenvolver uma compreensão ampliada da saúde, contribuindo para a construção de saberes transdisciplinares e inovação das práticas de atenção à saúde.

O modelo de atenção à saúde do SUS deve, para dar conta da situação de saúde brasileira, transformar-se em uma organização em redes de atenção à saúde. Para tanto, a primeira mudança deverá ser cultural e precisará rever o modelo hierárquico do SUS, expresso em sua organização por níveis de atenção - da atenção básica à média e alta complexidade. Na concepção de redes, a ideia de hierarquia deve ser substituída pela de poliarquia. Não há hierarquia entre os diferentes nós da rede sanitária: todos são igualmente importantes para os objetivos do sistema ${ }^{24}$. Entretanto, as redes de atenção à saúde apresentam uma característica singular: elas devem ter um centro de comunicação que coordene os fluxos das pessoas e das coisas na rede e que é constituído pela atenção primária à saúde, no Brasil chamado de atenção básica de saúde.

Repensar o modelo de assistência à saúde não é uma postura recente. Durante muito tempo ficou restrita a elaborações e reflexões realizadas por alguns profissionais que, atuando na assistência, passaram a perceber a importância do trabalho entre disciplinas ${ }^{25}$.

O grupo focal também destacou a relação entre universidade e serviços de saúde durante o processo de formação dos estudantes por meio de estágios. Essa dimensão também traz à tona a questão da inserção do nutricionista na Atenção Básica de Saúde-SUS. Para aprofundar o questionamento sobre o papel do SUS como política pública na garantia do acesso e acompanhamento à atenção nutricional da população brasileira diante das suas necessidades em saúde, é fundamental refletir sobre a perspectiva de inserção do nutricionista na rede de Atenção à Saúde do SUS.

O aluno aprende em NSP (disciplina Nutrição em Saúde Pública) como o nutricionista aborda os programas de saúde pública, tentando trazer para prática a teoria, mas quando o estudante chega na prática, ele não vê o nutricionista... . Surge 
aquele contraste em relação aos programas, como o SUS, onde os alunos veem tudo muito bonito no papel, mas quando chega na prática não encontra nada... . Falta inserir esse nutricionista nos programas... . Não há estrutura adequada para inserir o aluno no contexto que fica preso a um círculo vicioso: a prática tem pouco profissional pois a formação é frágil, a formação é frágil pois tem pouco profissional na prática (Discurso do Sujeito Coletivo - DSC).

Não se pode deixar de enfatizar que o paradigma atual de altas taxas de morbimortalidade por doenças crônicas não transmissíveis concretiza a necessidade do fortalecimento do nutricionista junto ao SUS. Perante essa complexa realidade social, é importante ressaltar que, em termos de saúde e nutrição, tanto o excesso de peso quanto a desnutrição devem ser tratados como componentes do mesmo processo. Do ponto de vista biológico, estudos já demonstraram que a desnutrição nos primeiros anos de vida aumenta o risco de excesso de peso na vida adulta. Do ponto de vista econômico e social, a maior prevalência de excesso de peso em famílias de menor renda pode também ser explicada pela estratégia adotada por esses grupos que privilegiam a compra de alimentos que proporcionem a maior quantidade de calorias pelo menor preço ${ }^{26}$.

A Atenção Básica é um terreno fértil para a implementação de ações de promoção da saúde e prevenção de doenças. O nutricionista é um profissional que deve ter seu perfil de atuação voltado para a melhoria das condições de saúde e nutrição da população brasileira ${ }^{1,8,14}$.

\section{O N CLUS Ã O}

A pesquisa proporcionou uma análise sobre a visão tanto de professores quanto de estudantes e recém-egressos do curso de Nutrição em relação às áreas de atuação do nutricionista, possibilitando desenvolver uma relação entre os principais fatores responsáveis pela situação atual do nutricionista na área de saúde pública.
Muito desafios precisam ser enfrentados para a melhoria do ensino de graduação na área de saúde, em especial de Nutrição. Entre eles, torna-se prioritário rever a perspectiva pedagógica tradicional e hegemônica que se baseia na lógica de transmissão vertical de conhecimento. As novas práticas pedagógicas devem fortalecer as dimensões de diálogo, trabalho interdisciplinar, problematização e análise critica ao longo da formação em Nutrição e Saúde Pública.

As inovações se constroem pelo reconhecimento de alternativas, saberes e práticas, nas quais se imbricam objetividade e subjetividade, saber popular e saber científico, teoria e prática, anulando dicotomias e gerando novos caminhos participativos e interdisciplinares. O processo de formação e trabalho em saúde precisa construir o novo perfil de formação de Nutrição em saúde pública: a ação profissional deve ser ampliada e estendida para o campo da segurança alimentar e nutricional.

\section{A GRADECIMENTOS}

Às alunas de graduação Jéssica Weber e Carolina Vogado pela contribuição durante a tabulação de resultado e ao nutricionista Douglas Moreira pelo auxílio na revisão final do artigo.

\section{OLABORADORES}

ARO PINHEIRO e E RECINE contribuíram na concepção e no desenho do projeto, análise e interpretação dos resultados, redação e revisão final do artigo. B ALENCAR contribuiu na análise e interpretação dos resultados, redação e revisão final do artigo. AA FAGUNDES e JS SOUSA contribuíram na elaboração do instrumento de coleta, na coleta e montagem do banco de dados. RA MONTEIRO e N TORAL contribuíram na concepção e desenho do projeto, e na redação do artigo.

\section{REFERÊ NCIAS}

1. Brasil. Ministério da Educação e Cultura. Conselho Nacional de Educação. Câmara de Educação Superior. Resolução CNE/CES n5. Institui Diretrizes Curriculares Nacionais do Curso de Graduação em 
Nutrição. Brasília: Ministério da Educação e Cultura; 2001.

2. Brasil. Câmara Interministerial de Segurança Alimentar e Nutricional. Plano Nacional de Segurança Alimentar e Nutricional: 2012/2015. Brasília: Ministério do Desenvolvimento Social e Combate à Fome; 2011.

3. Coutinho JGC, Gentil PC, Toral N. A desnutrição e obesidade no Brasil: o enfrentamento com base na agenda única da nutrição. Cad Saúde Pública. 2008; 24(2):S332-S40.

4. Vasconcelos FAG. O nutricionista no Brasil: uma análise histórica. Rev Nutr. 2002; 15(2):127-38. doi: 10.1590/S1415-52732000200001.

5. Ypiranga L, Gil MF. Formação profissional do nutricionista: por que mudar? In: Banduk MLS, Ruiz-Moreno L, Batista NA. A construção da identidade moral profissional na graduação de nutricionista. Interface. 2009; 13(28):111-20.

6. Gil MF. Recursos humanos em nutrição no Brasil: nutricionistas. Cad Saúde Pública. 1986; 2(4):561-9.

7. Vasconcelos, FAG. O nutricionista no Brasil: uma análise histórica. Rev Nutr. 2002; 15(2):127-38. doi: 10.1590/S1415-52732002000200001.

8. Brasil. Ministério da Educação e Cultura. Instituições de educação superior e cursos cadastrados [Internet]. [acesso 2011 abr 4]. Disponível em: $<$ http://emec.mec.gov.br/>.

9. Minayo MCS. O desafio do conhecimento: pesquisa qualitativa em saúde. 9a ed. São Paulo: Hucitec; 2006.

10. Anais do $21^{\circ}$ Congresso Brasileiro de Nutrição; 2010 26-29 maio; Joinville, SC. São Paulo: Associação Brasileira de Nutrição, 2010.

11. Lefreve F, Lafreve AM. Pesquisa de representação social: um enfoque qualiquantitativo, a metodologia do discurso sujeito coletivo. Brasília: Liber Livro; 2010. Série Pesquisa, 20.

12. Brasil. Ministério da Saúde. Pró-Saúde: Programa nacional de reorientação da formação profissional em saúde. Brasília: Ministério da Saúde; 2005.

13. Brasil. Ministério da Saúde. Portaria GM n¹54, de 24 de janeiro de 2008. Cria os núcleos de apoio à saúde da família. Brasília: Ministério da Saúde; 2008.

14. Brasil. Ministério da Saúde. Secretaria de Atenção à Saúde. Departamento de Atenção Básica. Política nacional de alimentação e nutrição. Brasília: Ministério da Saúde; 1999. Série B. Textos básicos de saúde.

15. Gambardella AMD, Ferreira CF, Frutuoso MFP. Situação profissional de egressos de um curso de nutrição. Rev Nutr. 2000; 13(1):37-40. doi: 10.15 90/S1415-527320000000100005.
16. Miranda DEGA, Pereira CHC, Paschoini TB, Quaglio T. O perfil de atuação dos ex-alunos do curso de nutrição de uma universidade do interior paulista. Investigação. 2010; 10(2-3):54-9.

17. Goulart AT. A importância da pesquisa e da extensão na formação do estudante universitário e no desenvolvimento de sua visão crítica. Horizonte. 2004; 2(4):60-73.

18. Banduk MLS, Moreno LR, Batista NA. A construção da identidade profissional na graduação do nutricionista. Interface. 2009; 13(28):111-20.

19. Portronieri FRDS, Elias RS, Fonseca ABC. A importância das disciplinas sociais a formação em nutrição na percepção dos alunos. Anais do $7^{\circ}$ Encontro Nacional de Pesquisa em Educação em Ciências; de 2009 nov. Brasil. Florianópolis: UFSC; 2009.

20. Bocchi CP. Segurança alimentar e nutricional no Orçamento e PPA [Internet]. Brasília: Consea; 2008 [acesso 2011 jun 10]. Disponível em: <http://www. planalto.gov.br/consea>.

21. Silva NF. O nutricionista e o acolhimento ao usuário do SUS nos NASF [Internet]. Brasília: Asbran; 2010 [acesso 2011 jun 22]. Disponível em: <http://www. asbran.org.br/>

22. Santos LAS, Silva MCM, Santos JM, Assunção MP, Portela ML, Soares MD, et al. Projeto pedagógico do programa de graduação em nutrição da Escola de Nutrição da Universidade Federal da Bahia: uma proposta em construção. Rev Nutr. 2005; 18(1): 105-17. doi: 10.1590/514115-52732005000100 010.

23. Buss PM, Pellegrini Filho A. A saúde e seus determinantes Sociais. Physis: Rev Saúde Col. 2007; 17(1):777-93.

24. Costa NMS. Formação pedagógica de professores de nutrição: uma omissão consentida? Rev Nutr. 2009; 22(1):97-104. doi: 10.1590/51415-52732 009000100009.

25. Ceccim RB. Equipe de saúde: a perspectiva entredisciplinar na produção de atos terapêuticos. In: Pinheiro R, Mattos RA. Cuidado as fronteiras da integralidade. São Paulo: Hucitec; 2004. v.10.

26. Brasil. Ministério da Saúde. Secretaria de Atenção à Saúde. Departamento de Atenção Básica. Coordenação-Geral da Política de Alimentação e Nutrição. Guia Alimentar para a População Brasileira: promovendo a alimentação saudável. Brasília: Ministério da Saúde; 2006. Série A. Normas e Manuais Técnicos.

Recebido em: 28/10/2011 Versão final em: 24/4/2012 Aprovado em: 25/6/2012 
